\title{
Total hip replacement in patients with history of illicit injecting drug use
}

\author{
Karl Wieser · Patrick O. Zingg • Michael Betz • \\ Georg Neubauer $\cdot$ Claudio Dora
}

Received: 30 December 2011/Published online: 4 April 2012

(C) Springer-Verlag 2012

\begin{abstract}
Background A history of illicit injecting drug use makes indication of total hip arthroplasty (THA) in patients with end stage hip osteoarthritis difficult, as the risk of infection with colonized strains is multiplied if the patient continues to inject or inhale illicit drugs.

Methods A retrospective survivorship analysis of a consecutive series of 27 THA in patients with a history of illicit drug use was performed. Follow-up evaluation consisted of (1) a WOMAC score, (2) a standardized interview including queries on drug habits and eventual additional medico-surgical treatments of the affected hip, (3) a clinical examination in order to complete a Harris Hip Score, (4) radiological examination and (5) blood tests (blood sedimentation rates and C-reactive protein). Defined endpoints were death, implant revised or awaiting revision for deep infection or any other reason and lost to follow-up or follow-up after at least 2 years.

Results Overall, 5- and 10-year implant survival rates with failure for any reason were $61 \%$ (CI: 41;81) and $52.3 \%$ (CI: 29;76) and for septic reasons $70.6 \%$ (CI: $52 ; 89)$ and $60.5 \%$ (CI: 36;85), respectively. Even if at the time of THA all patients and respective health care professionals confirmed abstinence of illicit injecting drug use, five patients reported occasional use. Declared abstinence of less than 1 year before THA was associated with higher recurrence rates $(p=0.001)$ and both with higher septic failure rates $(p=0.023, p=0.061)$. Positive serology for human deficiency virus did not increase implant failure rates.
\end{abstract}

K. Wieser $(\bowtie) \cdot$ P. O. Zingg · M. Betz · G. Neubauer · C. Dora Orthopaedic Department, Balgrist University Hospital, University of Zurich, Forchstrasse 340, 8008 Zurich, Switzerland e-mail: karl.wieser@balgrist.ch
Conclusion We use this unacceptable high failure rate as evidence when counseling patients and their health care professionals about the appropriate treatment of osteoarthritis in patients with a history of illicit drug use. Furthermore, we support the request of hair analysis for drugs documenting abstinence of at least 1 year before indicating THA.

Keywords Hip arthroplasty - Survivorship analysis . Illicit drug abuse

\section{Introduction}

Illicit injecting drug use has a long history, but it was in the early 1980s, with the rapid growth of intravenous heroine use and the spread of the human immunodeficiency virus (HIV), that this behavior gained prominence as a core element of Europe's and the world's drug problem. Today the estimated prevalence of injecting drug users is about 2.5 per 1,000 individuals aged 15-64 years, which corresponds to a total number of up to one million active injecting drug users in the 27 Member States of the European Union [1].

In developed countries, illicit injecting drug users have an increased risk of premature death from drug overdose, violence, suicide, alcohol-related causes and AIDS. Furthermore, the prevalence of infection with hepatitis $\mathrm{B}, \mathrm{C}$ and human immunodeficiency virus is estimated to be greater than $60 \%$ [2] and $12 \%$ [3], respectively. In HIV patients undergoing antiviral therapy, the risk of osteonecrosis of the femoral head (ONFH) is increased [4-6], with a prevalence of approximately $0.74 \%$ [4]. ONFH, together with inflammatory arthropathies due to repetitive bacteremia [7] and posttraumatic conditions are the main reasons for secondary hip osteoarthritis in illicit injecting drug users. 
The treatment strategy for illicit injecting drug users primarily aims to reduce drug injection-related harm and gradual reintegration of patients into normal working life and assimilation into the social community. Secondary hip osteoarthritis may sometimes compromise return to work and thus social reintegration. In such conditions orthopedic surgeons may be approached for adequate treatment of hip osteoarthritis. When total hip arthroplasty (THA) is anticipated, the risk of septic complications is a major concern, since the risk to cause and spread infection with colonized strains is known to be multiplied by injection or inhalation of drugs [8, 9]. On the other hand, alternatives such as hip arthrodesis or resection arthroplasty may hardly be perceived to give the highest attainable standard of care by patients and their supporting health care professionals, especially if promising efforts to stop illicit drug use have been made.

During the last decade it has been the practice of our institution to accept patients with a history of illicit injecting drug use for THA if they were in medical and social care by professionals specialized in drug-related problems and if abstinence from illicit drug use was declared by patients and confirmed by their respective healthcare professionals. It has been our impression that the septic complication rates were higher than expected from a normal population. Because of absence of any data on this issue, a retrospective review of all patients with a history of illicit drug use receiving THA in our department during the last decade was undertaken.

\section{Materials and methods}

Our prospectively collected database on THA was retrospectively analyzed for patients with a history of illicit injecting drug use undergoing THA between January 1999 and December 2009. From 2,625 primary THA performed during this time period, a consecutive series of 24 patients (27 THA), 17 male and 7 female, with an average of 43 years (range 28-55) was identified.

The study was approved by our institutional Human Subject Review Board. Written consent was obtained from all patients willing to attend a follow up visit. For those denying, based on a general permit issued by the responsible state agency, our institutional review board allows retrospective analysis of patient data relating to standard diagnostic or therapeutic procedures without individual informed consent. There was no external funding for the purpose of this study.

\section{Treatment protocol}

Before considering patients for THA, abstinence from illicit injecting drug use had to be declared by patients and confirmed by respective health care professionals and, in case of positive serology for hepatitis $\mathrm{C}$ virus (HCV) and/ or HIV, patients had to be followed by a healthcare institution specialized in infectious disease. Additionally, blood sedimentation rates (BSR) less than $35 \mathrm{~mm} / \mathrm{h}$ and C-reactive protein (CRP) levels less than $10 \mathrm{mg} / \mathrm{L}$ on preoperative blood screenings were mandatory.

Surgeries were performed under a laminar air flow system. Depending on the time period and underlying pathology, either a mini-incision direct anterior approach in the supine position (10 THA), or a transgluteal (14 THA) or transtrochanteric ( $3 \mathrm{THA}$ ) approach in the lateral position was used. Three doses of cefuroxime $1.5 \mathrm{~g}$ in an 8-h interval starting $30 \mathrm{~min}$ before incision and enoxaparin natrium on a daily dose for 6 weeks were given to all patients. Depending on the time period, non-cemented (14 THA) or hybrid (non-cemented cup and cemented stem) (13 THA) implants were used. One closed suction drain lying under the fascia was used and removed after $48 \mathrm{~h}$ in all cases.

\section{Follow-up evaluation}

All patients were invited by mail and asked for informed consent to participate in our review. Follow-up evaluation consisted of (1) a WOMAC score, (2) a standardized interview including queries on drug habits and eventual additional medico-surgical treatments of the affected hip, (3) physical examination looking for clinical signs of infection (open sinus, swelling, tenderness, warmth) in the hip region and in order to complete a Harris Hip Score (HHS), (4) radiological examination including an anteroposterior and cross-table lateral view, which were compared to the first postoperative X-rays and screened for radiolucent lines [10, 11], periosteal new bone formation and endosteal scalloping and (5) blood tests (BSR and CRP).

For patients not responding to our mail, our standardized interview was conducted by phone. For all patients a chart review recording all perioperative and postoperative complications, and in patients lost, an analysis of the latest available X-rays was performed. Defined endpoints were death, implant revised or awaiting revision for septic or any reason, lost to follow-up or follow-up after at least 2 years.

Septic failure was diagnosed if an open sinus, an abscess formation, frank pus during surgery or positive intraoperative culture from a subsequent surgical intervention were recognized. Additionally, deep sepsis was suspected if at least three of the following criteria were present: (1) erythematous, swollen, fluctuating and tender surgical site, (2) fever, chills or sweating, (3) old closed sinus, (4) elevated BSR $>35 \mathrm{~mm} / \mathrm{h}$ and $\mathrm{CRP}>10 \mathrm{mg} / \mathrm{L}$ in the absence of recent surgery, neoplasia, remote infection or other inflammatory conditions, (5) periosteal new bone 
formation with or without endosteal scalloping or early rapidly progressive radiolucent lines and (6) positive joint aspiration.

\section{Statistical analysis}

Kaplan-Meier survivorship curves were compiled with implant failure for septic and for any reason as endpoints, respectively. For comparisons of categorical data between groups a two sided Fisher Exact Probability Test was used. All statistical analyses were performed using SPSS 17.0 for Windows. The significance level was set at $p<0.05$.

\section{Results}

Indications for THA are summarized in Table 1. Seven patients had previous surgery on the affected hip a mean of 6.6 years (range 0.3-31; SD 12.1) before the index THA. Among these were two patients who had resection arthroplasty because of septic arthritis before. The course of all patients is summarized in Table 2.

Six patients (8 THA's) were lost to follow-up or deceased

Two of them (3 THA's) left for a foreign country and could not be traced. One, left after septic implant revision (Escherichia coli) 2 months after the index procedure, was included in survivorship analysis. The other four patients (5 THA's) died after a mean postoperative time of 24 months (range 6-73, SD 33) unrelated to the surgery. Three had an uneventful postoperative course. And one had a septic revision 5 weeks after the index procedure (coagulase negative Staphylococcus).

Four patients with telephone interview and chart review

Four refused a follow-up visit but telephone interview and chart review revealed an uneventful course in two (24 and 98 months after THA) and septic revision in two, 24 (Staphylococcus aureus) and 69 months (coagulase negative Staphylococcus) after the index procedure.

Table 1 Indications for THA

\begin{tabular}{lr}
\hline Primary OA & 7 \\
Secondary OA & 10 \\
ONFH & 2 \\
Postinfectious OA & 6 \\
Posttraumatic OA & 2 \\
Childhood's disease & \\
\hline
\end{tabular}

Fourteen patients available for follow-up visit

After an average time of 69 (range 24-123; SD 38) months, nine patients ( 9 THA's) had an uneventful course, without any clinical signs of infection and perfect radiological appearance of the THA. CRP levels and ESR were normal in seven of them. Two had elevated values for CRP (25 and 19) and BSR (38 and 29), one after a recent pneumonia, and the other without detectable reason. There overall mean HHS and WOMAC were 91 (range 71-100) and 0.8 (range $0-2$ ), respectively.

Two patients sustained a traumatic periprosthetic fracture. One Vancouver type B2 fracture was recognized 11 months after the index procedure when loosening of the implant together with a non-union was observed. Intraoperative tissue specimens sent for microbiological examination were negative. The other (Vancouver type B2) fracture was due to a road traffic accident 6 months after index procedure. This was treated with a long revision stem in a foreign country; however this was complicated with a postoperative deep infection (Staphylococcus aureus). Both showed normal values for CRP and ESR at follow-up examination. HHS (99 and 100 points) and WOMAC scores ( 0 and 0 points) were excellent.

One patient was revised due to periprosthetic infection (coagulase negative Staphylococci) 1 month after index procedure. Treatment consisted of a one-stage implant revision and ciprofloxacin and rifampicin for 3 months. At follow-up, 27 months after the index procedure, no local signs of infections were encountered and $\mathrm{X}$-rays were excellent. CRP measured 16 and ESR $25 \mathrm{~mm} / \mathrm{h}$. HHS was 89 and the WOMAC 0 points.

One patient with bilateral THA showed 30 months after index surgery elevated CRP (26) and BSR (52) levels as well as implant loosening with gross metaphyseal and acetabular osteolysis on both sides (Fig. 1), but denied further investigation such as hip aspiration for bacteriological tests. However, due to clinical and radiological findings, both hips were considered as septic failures.

One patient underwent surgical implant revision due to deep infection with a total of three two-stage exchanges because of recurrent infections, first with $S$. aureus 6 months after the index procedure, second with Streptococcus mitis and third with Streptococcus sanguis and Candida albicans. A resection arthroplasty was finally performed.

Overall, from 25 THA (in 23 patients) followed until implant failure, death or for a minimum of 24 months, 8 were revised and 2 are awaiting revision. As a cause of failure, deep sepsis was detected in 8 cases. Five- and 10-year implant survival rates with failure for any reason were $61 \%$ (CI: $41 ; 81$ ) and $52.3 \%$ (CI: 29;76) and for septic reasons $70.6 \%$ (CI: 52;89) and $60.5 \%$ (CI: 36;85), respectively. Figure 2 shows Kaplan-Meier survivorship analysis. 


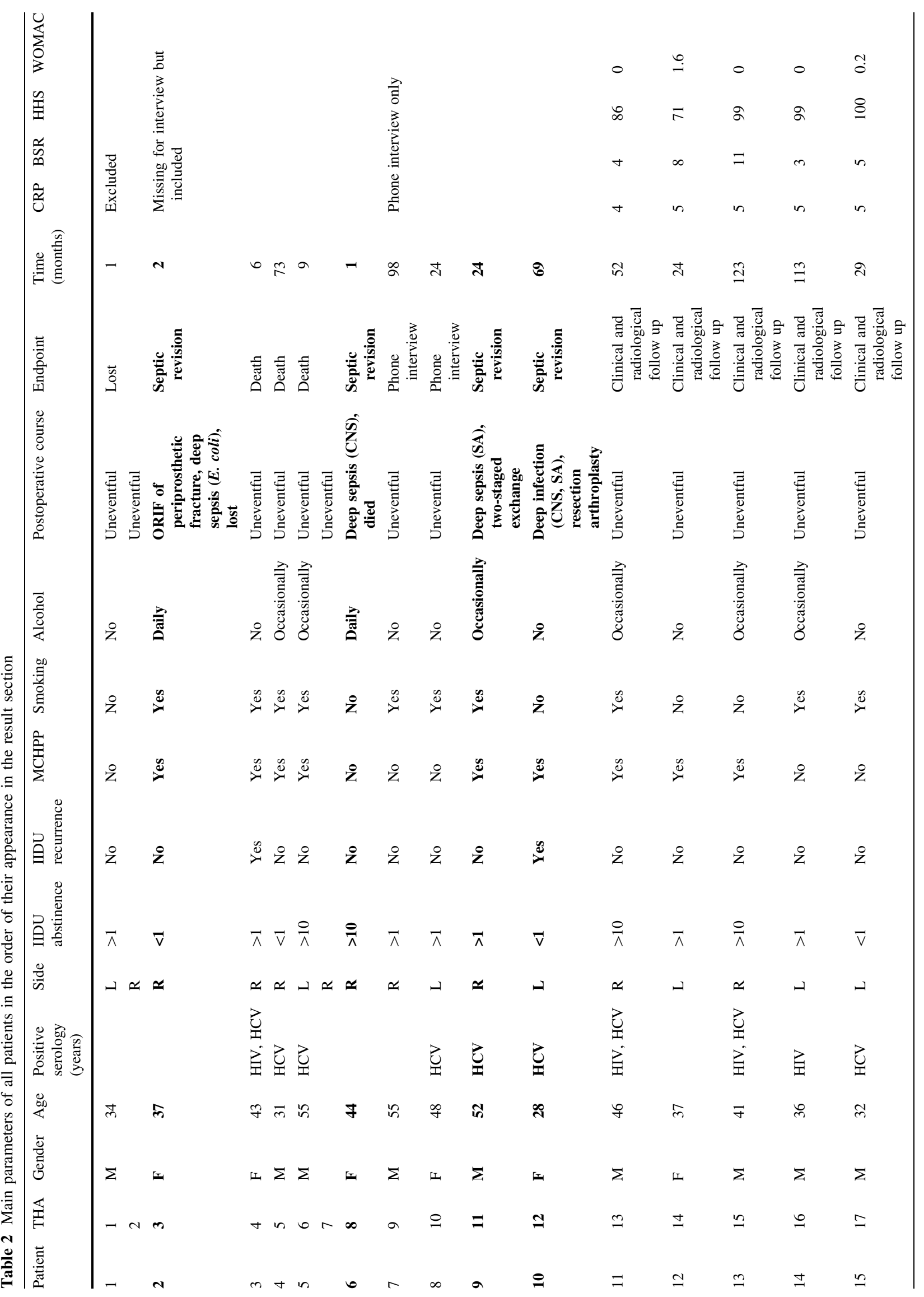


Arch Orthop Trauma Surg (2012) 132:1037-1044

1041

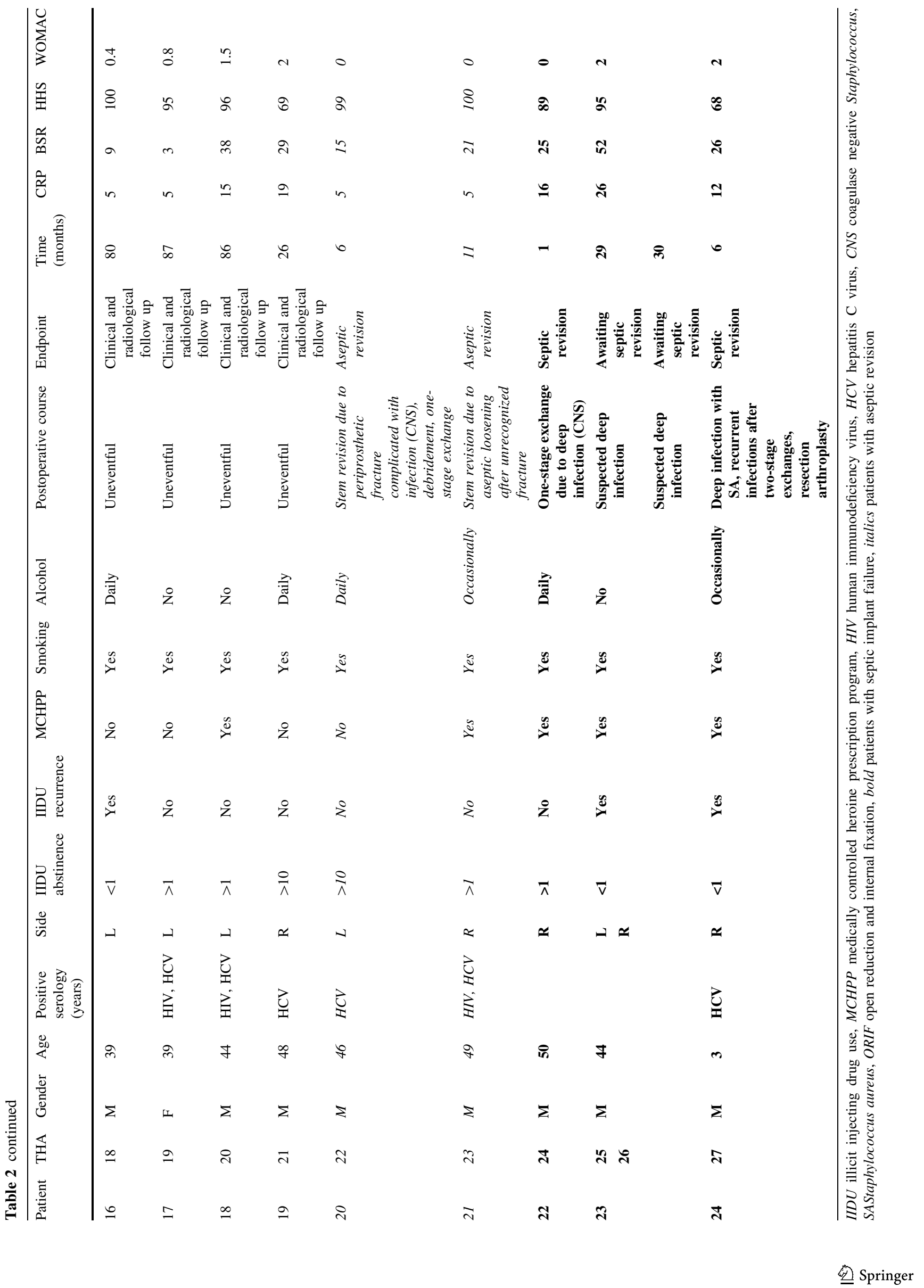



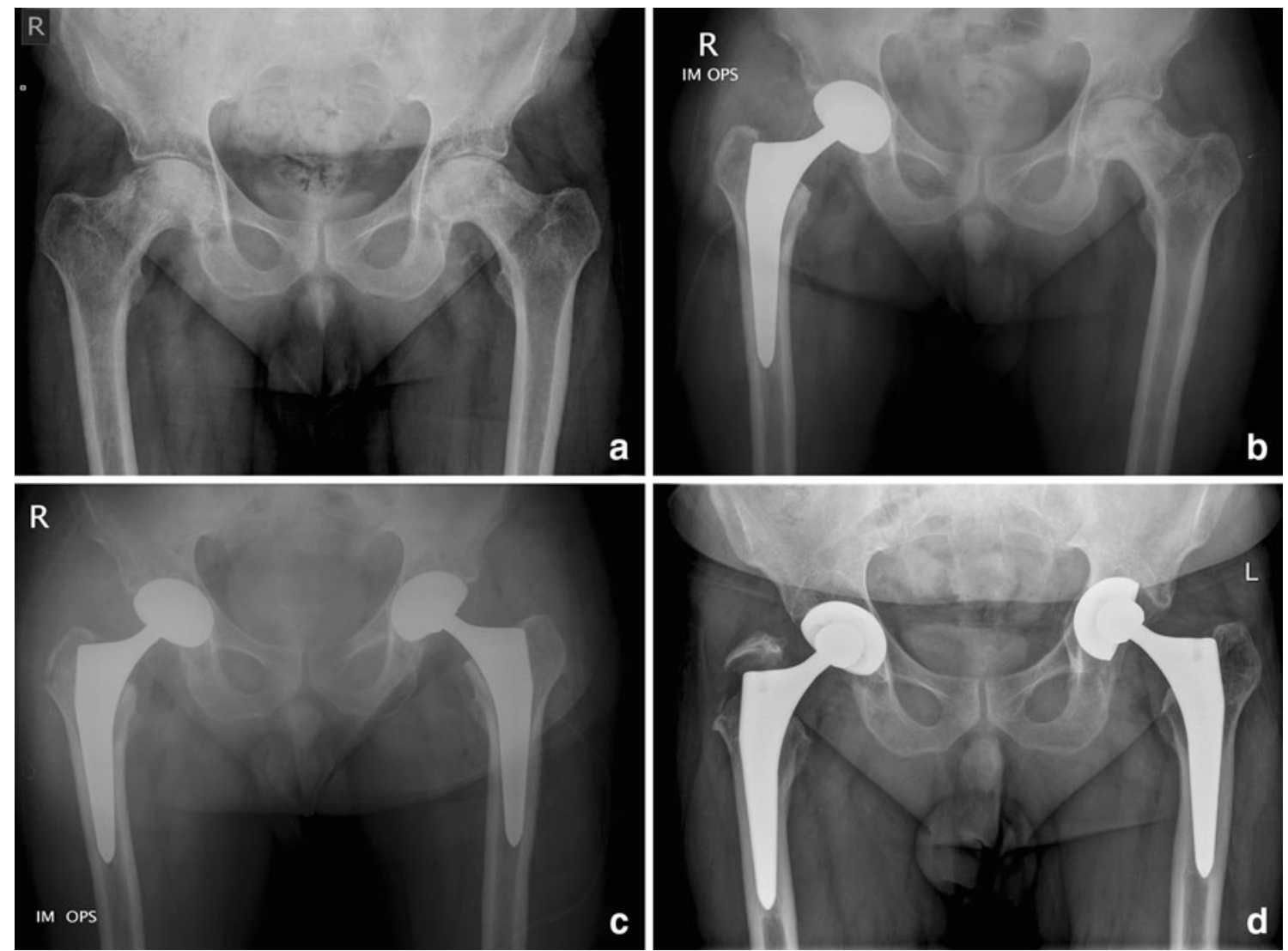

Fig. 1 X-ray of a 44-year-old man with bilateral osteonecrosis of the femoral head (a). A minimal invasive total hip arthroplasty was performed first on the right (b) and 6 weeks later on the left side (c). Postoperative X-rays showed a non-symptomatic avulsion fracture of the greater trochanter on the right side, which was treated

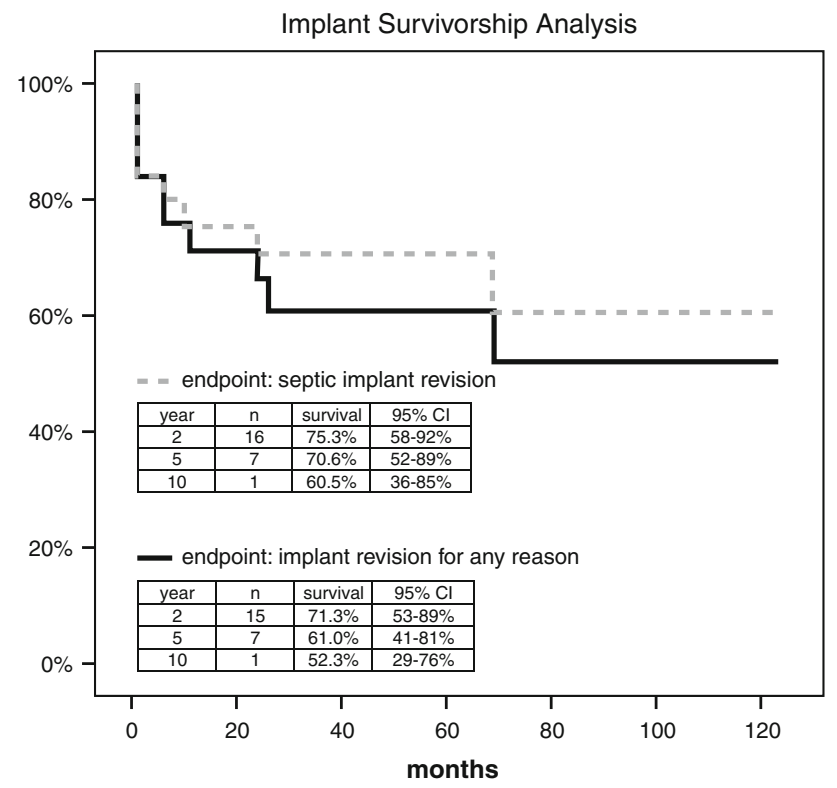

Fig. 2 Kaplan-Meier survivorship analysis with implant failure for septic and for any reasons as endpoints conservatively. 30 months after index procedure the patient presented elevated BSR and CRP levels as well as implant loosening, cup dislocation and gross metaphyseal and acetabular osteolysis on both sides (d). Although he denied joint aspiration and any kind of surgical treatment, both hips were considered as septic failures

According to our chart review, abstinence from illicit injection drug use at time of the index surgery was declared to be greater than 10 years in six, greater than 1 year in ten and less than 1 year in eight patients. Fourteen participated in a medically controlled heroine prescription program. The others were abstinent from illicit drug use without substitution. In 5 of 24 patients, recurrence of illicit injecting drug use was recorded either from the questionnaire or from the charts. All recurrences occurred in patients having stopped illicit drug use for less than 1 year before the index surgery $(p=0.001)$ and four of them participated in a medically controlled heroine prescription program $(p=0.36)$.

Four septic failures out of 5 THA's occurred in patients admitting illicit drug use recurrence and 3 septic failures out of 20 THA's in patients not admitting illicit drug use recurrence ( $p=0.023$ ). Five septic failures out of 8 THA's occurred in patients having stopped illicit drug use for less than 1 year and 3 out of 14 THA's in patients having stopped illicit drug use for more than 1 year $(p=0.061)$. 
Seven patients had positive serology for HIV, 16 for HCV and 6 for both, all under a medically supervised treatment protocol. None of the THA's implanted in HIVpositive patients were revised because of deep sepsis.

At time of index surgery 19 patients were smoking at least ten cigarettes a day, 6 admitted to daily smoking and further 8 occasionally abused alcohol.

\section{Discussion}

In this retrospective analysis the 10-year cumulative septic implant revision rate of THA in patients with a history of illicit injecting drug use was as high as $39.5 \%$ (CI: 15;64) and verified our concerns about an unacceptable high risk of septic complications in this population.

During the last decade, $1 \%$ of primary THA's were performed in patients with a history of illicit injecting drug abuse in our institution. This is more than would be expected when considering the approximately 30,000 illicit injecting drug users in our country, representing about $0.4 \%$ of our population. The urban location of our department and our practice to accept patients with a history of illicit injecting drug use for THA may explain this higher than expected rate.

With a mean age of 43 years, our patient population represents a relatively young group undergoing THA. This is most likely attributable to the etiology of osteoarthritis being femoral head osteonecrosis and septic arthritis in nearly half of the cases, which both are considered typical hip-specific complications of illicit injecting drug abuse and HIV.

Despite this young patient collective, four of 23 patients died during the follow-up period. Injecting drug use related deaths primarily occur among young adults and accounts for a large number of life years lost in developed societies. In Scotland and Spain for example, injecting drug related deaths accounts for 25-33\% of deaths of young adult males.

The high percentage of patients with positive serology for HIV (29 \%) and HCV (54 \%) is a known association in patients with illicit injecting drug use and can be explained by the sharing of syringes and other injection equipments. All HIV seropositive patients were under treatment of antiviral therapy and followed by the local department of infections disease and none had symptoms of acquired immune deficiency syndrome.

Most disappointing was the $47.7 \%$ implant failure rate at 10 years, $39.5 \%$ due to deep sepsis. We are not aware of any other series that have reported the outcomes in a similar patient population; hence our results could be compared.

Looking to non-hemophilic HIV-positive patients, a higher rate of septic complications can yet not be concluded from the literature [12-16]. However, THA in hemophilic HIV seropositive patients is indeed associated with a high septic revision rate, which in Hicks's series [12] was as high as $18.7 \%$ in primary and $36.4 \%$ in revision THA's. In one of these series [16] a subgroup of 5 HIV-positive illicit injecting drug users was included and 3 of them were complicated by deep sepsis after $1,1.5$ and 15 months. In our present series none of the patients sustaining deep sepsis was HIV seropositive and HIV therefore does not explain the high revision rate.

The risk to cause and spread infection with self-colonized strains has shown to be multiplied by injection or inhalation of drugs [8, 9]. Furthermore, the rate of periprosthetic joint infection after documented Staphylococcus aureus bacteremia is reported to range from 30 to $40 \%$ [17]. It is, therefore, not surprising that injecting drug users more often sustain septic complications after THA's. In the present series, all patients affirmatively stated and their respective healthcare professionals confirmed abstinence from illicit injecting drug use at the time of the index procedure. Nevertheless, five patients admitted occasional recurrence of illicit injecting drug use after the index procedure. Abstinence of less than 1 year before index procedure was significantly associated with recurrence of illicit injecting drug use and both with septic failure of the prosthesis. However, if the patients with recurrence of illicit drug abuse were excluded, the septic failure rate would still reach $25 \%$. Although other risk factors for septic failure, such as younger age [18], alcohol abuse [19] and heavy smoking [20] were present in our group of patients, this still does not explain the exceedingly high complication rate.

From our data, we are unable to elucidate whether this high septic failure rate was due to the altered general health status of patients, who recently stopped injecting drug use thus making them more susceptible to infection or whether these patients are simply at higher risk of relapse, which they may not admit. In our study, drug tests [21, 22] in order to verify abstinence from illicit drug use were not performed. Additional limitations of the present study are its retrospective nature with difficulties of complete followup, the small group of patients with heterogenic indications for THA and different co-morbidities limiting statistical analysis. Furthermore, one can debate whether the origin of periprosthetic infection was hematogenous as opposed to an exogenous.

Despite these limitations valuable conclusions can be made: (1) THA's in patients with declared abstinence after a history of illicit injecting drug use results in an unacceptable high septic failure rate. (2) Declared abstinence of less than 1 year before THA is more often associated with recurrence of illicit drug abuse and both increase the risk of septic implant failure. 
Therefore, from this study we have changed our current practice and have adopted the results as evidence when negotiating appropriate treatment of osteoarthritis with patients and their supporting health care professionals. We are also requesting hair analysis for drugs, documenting abstinence of at least 1 year before indicating THA.

\section{References}

1. European Monitoring Centre for Drugs and Drug Addiction (2010) Trends in injecting drug use in Europe. Publications office of the European Union, Luxembourg. ISBN:978-92-9168-412-0

2. Garfein RS, Vlahov D, Galai N, Doherty MC, Nelson KE (1996) Viral infections in short-term injection drug users: the prevalence of the hepatitis $\mathrm{C}$, hepatitis B, human immunodeficiency, and human T-lymphotropic viruses. Am J Public Health 86:655-661

3. March JC, Oviedo-Joekes E, Romero M (2007) Factors associated with reported hepatitis $\mathrm{C}$ and HIV among injecting drug users in ten European cities. Enferm Infecc Microbiol Clin 25:91-97

4. Yombi JC, Vandercam B, Wilmes D, Dubuc JE, Vincent A, Docquier PL (2009) Osteonecrosis of the femoral head in patients with type 1 human immunodeficiency virus infection: clinical analysis and review. Clin Rheumatol 28:815-823

5. Hensgens MP, Mudrikova TT, van der Meer JT (2009) Osteonecrosis as late complication in HIV-infected patients. Ned Tijdschr Geneeskd 153:B382

6. Eholié SP, Ouiminga M, Ehui E, Nzunetu G, Ouattara SI, Konan AV, Anglaret X, Bissagnéné E (2009) Avascular osteonecrosis of the femoral head in three West African HIV-infected adults with heterozygous sickle cell disease. Antivir Ther 14:1011-1014

7. Lamas JL, Ocampo A, Martínez-Vázquez C, Miralles C, Longueira R, Arias M, Sopeña B, Rivera A, Portela D (2010) Asymptomatic osteonecrosis of the hip in HIV-infected patients. Enferm Infecc Microbiol Clin 28:150-155

8. Gordon RJ, Lowy FD (2005) Bacterial infections in drug users. N Engl J Med 353:1945-1954

9. Lowy FD, Miller M (2002) New methods to investigate infectious disease transmission and pathogenesis-Staphylococcus aureus disease in drug users. Lancet Infect Dis 2:605-612
10. Gruen TA, McNeice GM, Amstutz HC (1979) "Modes of failure" of cemented stem-type femoral components: a radiographic analysis of loosening. Clin Orthop Relat Res 141:17-27

11. DeLee JG, Charnley J (1976) Radiological demarcation of cemented sockets in total hip replacement. Clin Orthop Relat Res 121:20-32

12. Hicks JL, Ribbans WJ, Buzzard B, Kelley SS, Toft L, Torri G, Wiedel JD, York J (2001) Infected joint replacements in HIVpositive patients with haemophilia. J Bone Joint Surg $\mathrm{Br}$ 83:1050-1054

13. Parvizi J, Sullivan TA, Pagnano MW, Trousdale RT, Bolander ME (2003) Total joint arthroplasty in human immunodeficiency virus-positive patients: an alarming rate of early failure. J Arthroplasty 18:259-264

14. Harrison WJ, Lavy CB, Lewis CP (2004) One-year follow-up of orthopaedic implants in HIV-positive patients. Int Orthop 28:329-332

15. Harrison WJ (2005) HIV/AIDS in trauma and orthopaedic surgery. J Bone Joint Surg Br 87:1178-1181

16. Habermann B, Eberhardt C, Kurth AA (2008) Total joint replacement in HIV positive patients. J Infect 57:41-46

17. Sendi P, Banderet F, Graber P, Zimmerli W (2011) Periprosthetic joint infection following Staphylococcus aureus bacteremia. J Infect 63:17-22

18. Malinzak RA, Ritter MA, Berend ME, Meding JB, Olberding EM, Davis KE (2009) Morbidly obese, diabetic, younger, and unilateral joint arthroplasty patients have elevated total joint arthroplasty infection rates. J Arthroplasty $24: 84-88$

19. Tomás $\mathrm{T}$ (2008) Patient-related risk factors for infected total arthroplasty. Acta Chir Orthop Traumatol Cech 75:451-456

20. Espehaug B, Havelin LI, Engesaeter LB, Langeland N, Vollset SE (1997) Patient-related risk factors for early revision of total hip replacements. A population register-based case-control study of 674 revised hips. Acta Orthop Scand 68:207-215

21. Liniger B (2011) The value of laboratory analysis for general practitioners assessing fitness to drive of persons suspected problems with alcohol, illicit drugs or medicaments. Ther Umschau 68:265-268

22. Balikova M (2005) Hair analysis for drugs of abuse. Plausibility of interpretation. Biomed Pap Med Cac Univ Palcky Olomouc Czech Repub 149:199-207 